\title{
Early Detection System of Harmful Algal Bloom Using Drones and Water Sample Image Recognition
}

\author{
Fukuyoshi Kimura, ${ }^{1,2 *}$ Akihiro Morinaga, ${ }^{2}$ Masayoshi Fukushima, ${ }^{3}$ \\ Tomonari Ishiguro, ${ }^{3}$ Yasuhiko Sato, ${ }^{1}$ Akihiro Sakaguchi, ${ }^{4}$ \\ Tomoyuki Kawashita, ${ }^{4}$ Ikuo Yamamoto, ${ }^{2}$ and Toru Kobayashi ${ }^{2}$ \\ ${ }^{1}$ System Five Co. Ltd., 1-9-31 Hayama, Nagasaki-shi, Nagasaki 852-8053, Japan \\ ${ }^{2}$ Graduate School of Engineering, Nagasaki University, \\ 1-14 Bunkyomachi, Nagasaki-shi, Nagasaki 852-8521, Japan \\ ${ }^{3}$ KDDI Corporation, 3-10-10 Iidabashi, Chiyoda-ku, Tokyo 102-8560, Japan \\ ${ }^{4}$ Department of Control Engineering, National Institute of Technology, Sasebo College, \\ 1-1 Okishinmachi, Sasebo-shi, Nagasaki 857-1192, Japan
}

(Received April 29, 2019; accepted August 6, 2019)

Keywords: image recognition, harmful algal bloom, drone, web application, convolutional neural network $(\mathrm{CNN})$

Food consumption is increasing as the world population increases. While the eating of fish is spreading worldwide, the depletion of fishery resources has become a problem owing to overfishing, and the importance of aquaculture is increasing in order to continue to supply fish as food. In marine aquaculture, fish are grown in aquaculture cages in the sea. Thus, if a harmful algal bloom (HAB) reaches the cages, it will cause serious damage. Countermeasures against $\mathrm{HAB}$ are one of the important problems for aquaculture fishermen. In Nagasaki Prefecture in Japan, countermeasures against HAB include patrolling and sampling water by ships. These samples are then submitted to HAB experts for analysis. Following this analysis, notification is provided to aquaculture fishermen. When HAB is detected early, aquaculture fishermen can minimize the damage of HAB by stopping feeding and moving the aquaculture cages. In this study, we developed a system of early detection and notification of HAB to aquaculture fishermen. This is carried out by patrolling and sampling water using drones, detection of $\mathrm{HAB}$ by microscope and $\mathrm{PC}$ operation, and automatic notification by email and web application. As a result of this developed system, the identification accuracy of harmful plankton is more than $90 \%$, and the time taken to find HABs can be shortened from the $6 \mathrm{~h}$ of the conventional approach to $15 \mathrm{~min}$ using this newly developed system.

\section{Introduction}

According to a presentation to the United Nations, the world population will reach 9.7 billion in $2050 .^{(1)}$ Food consumption is also increasing with the population. While the eating of fish is increasing worldwide, depletion of fishery resources has become a problem owing to overfishing, and the importance of aquaculture is increasing in order to continue to supply fish

*Corresponding author: e-mail: f-kimura@sfk-nga.co.jp

https://doi.org/10.18494/SAM.2019.2417 
as food. ${ }^{(2)}$ As natural fish that inhabit the sea are limited resources, in recent years the amount of catch has been limited for each fish type to prevent the depletion of the resources, and it is difficult to increase the amount of catch of natural fish more than what it currently is. In order to supply fish sustainably in the future, it is necessary to develop aquaculture. There are two major types of aquaculture; marine aquaculture and land aquaculture. In marine aquaculture, fish are grown in aquaculture cages in the sea, so if a harmful algal bloom (HAB) reaches the cages, it will cause serious damage. Countermeasures against HAB are one of the important issues for aquaculture fishermen. When an algal bloom occurs, it is also important to determine whether the algal bloom contains harmful plankton.

In Nagasaki Prefecture in Japan, countermeasures against HAB include patrolling and sampling water by ships. These samples are then submitted to HAB experts for analysis. Subsequently, the results of the analysis are provided to the aquaculture fishermen. When HAB is detected early, aquaculture fishermen can minimize the damage due to HAB by stopping feeding and moving the aquaculture cages.

In cases of existing systems for detecting algal bloom, the occurrence of algal bloom is detected by continuously observing the state of the ocean using multiple fixed-point observation sensors or web-based geographical information systems. ${ }^{(3,4)}$ On the other hand, in recent years, systems for sampling water by drone has been developed. ${ }^{(5,6)}$ However the drone systems are not reported to be able to adjust the sampling water depth. As harmful plankton is often distributed deeper than one meter in the sea, it is important to sample water at some depths. Regarding plankton image recognition in microscopic images, $\mathrm{Hu}$ and Davis reported that after extracting the feature quantities of plankton images, support vector machines classified plankton into seven types; the accuracy rate was $72 \%$. ${ }^{(7)}$ Zheng and Wang reported a $75.726 \%$ accuracy rate using a system that classified 121 plankton type images using convolutional neural network $(\mathrm{CNN}){ }^{(8)}$

In this study, we aimed to develop a system that enables early detection and early countermeasures against $\mathrm{HAB}$ in a shorter time than the conventional method. Figure 1 shows

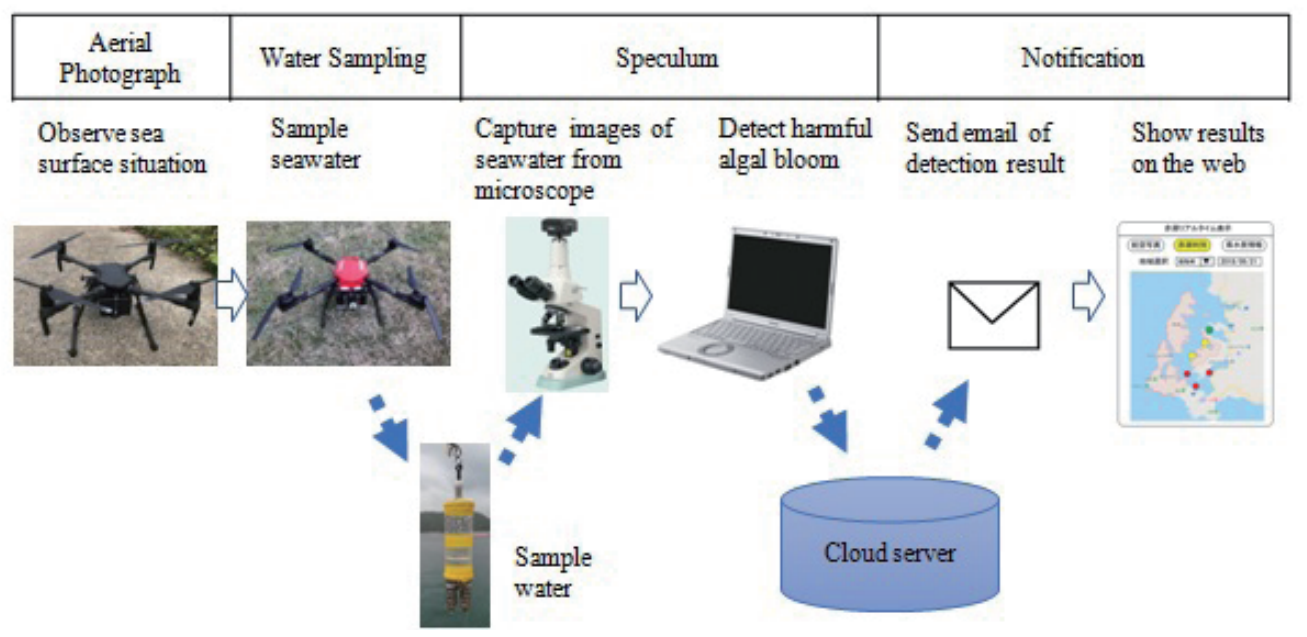

Fig. 1. (Color online) Overview of the system. 
an overview of the system. There are three main phases in the constructed system. First, an aerial photography drone monitors the sea surface to identify anomalies in the sea surface color, and then a water sampling drone samples the seawater. Drones can move faster than ships, and require relatively little additional skill to operate. Next, microscopic images of seawater are input to a computer, the images are recognized and classified into types of plankton, the number of plankton cells is counted, and the occurrence of HAB is determined. Since the PC recognizes types of plankton, the person placing the seawater sample in the microscope does not need specialized knowledge to be able to obtain results. Finally, the aquaculture fishermen are notified of the detection results by email, and receive HAB alert information via a web application. Storing the results of the examination on the server will enable automatic notification by email, and will also allow the user to check the status using a web application. As a result of this developed system, the identification accuracy of harmful plankton is more than $90 \%$, and the time taken to find HABs can be shortened from the $6 \mathrm{~h}$ required with the conventional approach to $15 \mathrm{~min}$.

In Sect. 2, we describe the sea surface aerial photography and water sampling drone systems, in Sect. 3, the HAB detection system, and in Sect. 4, the real-time notification system.

\section{Sea Surface Photography and Seawater Sampling Drone System}

In this section, we describe the sea surface aerial photography drone and seawater sampling drone system for sampling seawater from the area of suspected HAB occurrence.

\subsection{Aerial photography drone}

In the conventional method, water sampling for analysis was performed at predetermined fixed points or when signs of algal bloom are observed on the sea surface by ship patrols. However even if signs of algal bloom are observed, it takes a significant amount of time to observe large areas of the sea, and as it is impractical to carry out exhaustive observation, there is always the risk that an important area may be overlooked.

In our system, the sea surface is photographed from the sky by a drone, and signs of algal bloom are detected by comparing the color conditions photographed with a reference color sample. By using the drone, it is possible to monitor a wide area from a high altitude in a short time and to reduce the cost due to fuel, for example, compared with ships. The aerial photography drone used in this system is Matrice 200 from DJI and is equipped with ZENMUSE Z30 from DJI as the aerial camera that can capture detailed images of the sea surface from a high altitude.

\subsection{Seawater sampling drone}

When signs of algal bloom are detected by the aerial photography drone, its location information is sent to the base station. Then, seawater is sampled using the seawater sampling drone in order to confirm, by means of a microscope, whether harmful plankton is actually 
present. The seawater sampling drone named Akabot II was developed during this study. The drone is a quadcopter and employs a DJI flight controller. This quadcopter is equipped with a water sampling device that is connected to a pulley attached to the body by a wire, and it is possible to lower and raise the water sampling device by remote operation. As shown in Fig. 2, the water sampling device consists of three bottles in which the valve automatically opens at a given water pressure. This preset opening water pressure makes it possible to collect seawater from a predetermined depth by adjusting the length of a spring using a bolt in advance. Figure 3 shows a photograph of a flying drone lowering a sampling bottle into the sea. It is possible to sample water from three points or three depths $(1,3$, and $5 \mathrm{~m})$ in one flight. Furthermore, by taking a photograph using a camera that includes GPS data when lowering a bottle into the sea, it is possible to confirm the point at which the water sampling was performed after the flight.

\section{HAB Detection System}

In this section, we describe the $\mathrm{HAB}$ detection system that take pictures from a microscope, classifies the extracted images by plankton type, counts the number of cells of each plankton type, and determines the risk level of the HAB.

\subsection{Configuration of $\mathrm{HAB}$ detection system}

Around the coasts of Nagasaki Prefecture in Japan, algal blooms occur several times each year and contains various types of plankton. Among them, there are five types of plankton that cause particularly serious damage. These five types of plankton are cultured for research at the Nagasaki Prefectural Institute of Fisheries. Therefore, we focused on the detection of these five harmful planktons that cause HABs, using our system. In the detection of HABs using seawater samples, conventionally, a person with expert knowledge of plankton observes the form of plankton with a microscope, classifies the type, and counts the occurrence of each type. In the proposed system, since the classification of plankton is performed by image recognition using a CNN, counting of each plankton type possible even if people do not have expert knowledge of plankton.

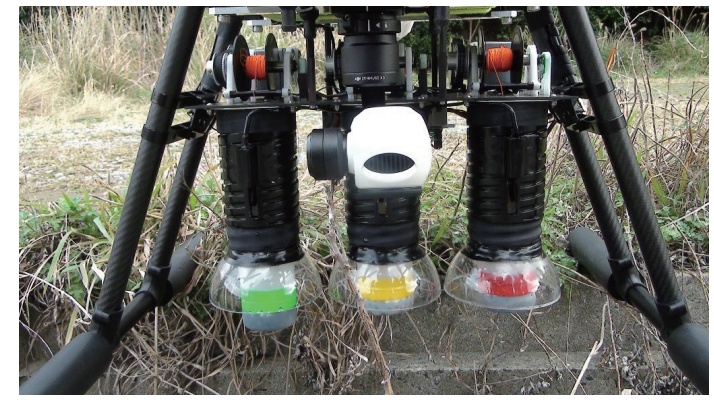

Fig. 2. (Color online) Three sampling bottles.

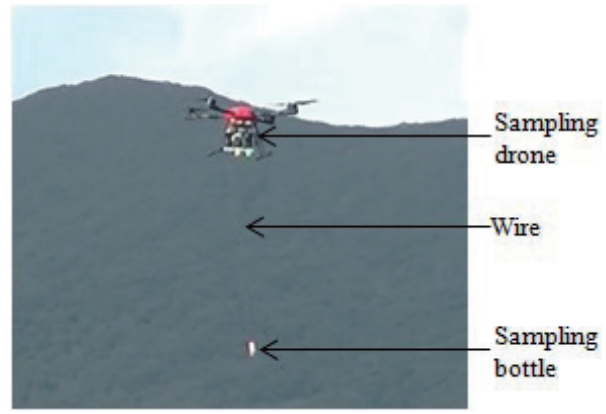

Fig. 3. (Color online) Lowering the sampling bottle. 
The flow of operations for detecting the occurrence of HAB using the HAB detection system is shown in Fig. 4. The user receives the seawater sampled by the drone, and sets the seawater for analysis on the microscope. After the evaluation area is adjusted on the microscope, the image of the target area for evaluation is input to the PC via the USB of the camera attached to the microscope. Harmful plankton is detected and the number of cells of harmful plankton is counted for each plankton type by image recognition. The result is displayed on the PC screen, and the data is stored on a cloud server.

The flow of processing by PC is shown in Fig. 5. From the input microscope images, images of individual target objects are extracted. The extracted images are classified into plankton types. The number of cells of each plankton type are counted and the densities of these are calculated. The risk of HAB is determined on the basis of the densities.

\subsection{Extraction of plankton images}

We describe the process of identifying the plankton from each image taken by the camera attached to the microscope. When the seawater sampled by drone is received, $0.3 \mathrm{~mL}$ of the seawater is injected into the plankton counter plate. Then the plankton counter plate is set on the microscope. Images are input to the PC via the camera attached to the microscope. Table 1 shows the imaging conditions of the camera attached to the microscope.

From the image of the entire observation area, areas targeted for image recognition are identified. Since the images are observed by bright field observation with a transmission microscope, the background is bright and the objects such as plankton are dark. We considered binarization processing by image brightness to be a suitable method for distinguishing the background and target object. As shown in Fig. 6(a), an image of the observation area is converted to grayscale and binarized to separate the target object. As binarization methods, a discriminant analysis method and binarization with an adaptive threshold were compared, and the latter was adopted. Binarization with adaptive thresholding is excellent in that separation can be performed even when there is light and shade variation in the background. ${ }^{(9-11)}$ For

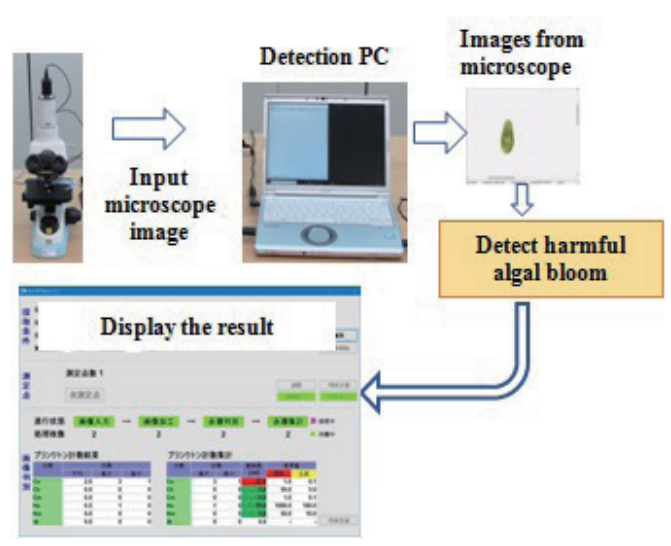

Fig. 4. (Color online) System configuration.

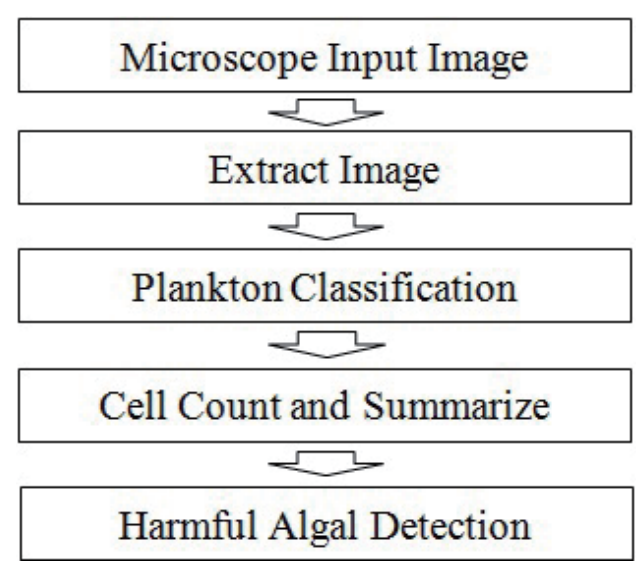

Fig. 5. Flow of image processing. 
Table 1

Microscope camera setting.

\begin{tabular}{lc}
\hline Object lens magnification & 10 \\
Exposure Time (ms) & 10 \\
Image resolution & $2048 \times 1536$ \\
Clock $(\mathrm{MHz})$ & 38 \\
\hline
\end{tabular}

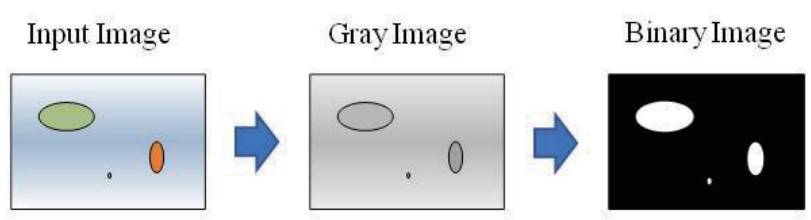

(a)

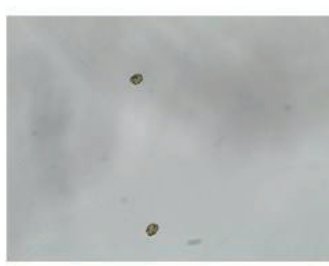

(b)

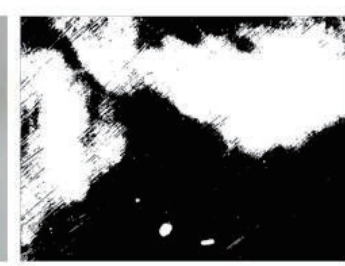

(c)

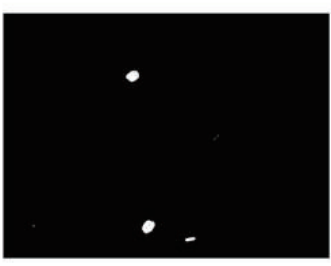

(d)

Fig. 6. (Color online) Results of discriminant analysis method and binarization with adaptive threshold. (a) Input to binary image, (b) input image, (c) discriminant analysis method, and (d) adaptive threshold.

example, as shown in Fig. 6(b), comparison was made in the case where an image in which there are two individual plankton is input. Figure 6(c) is an image that has been binarized by discriminant analysis, and Fig. 6(d) is an image that has been binarized by adaptive thresholding. In both cases, the black and white reversal process is performed after the binarization process. In discriminant analysis [Fig. 6(c)], one of the plankton types may be obscured by the shadow of the background. The adaptive threshold [Fig. 6(d)] clearly separates the two types of plankton from the background. Similarly, for the 20 images in which the plankton and background cannot be clearly separated by the discriminant analysis method, separation was possible as a result of verifying the binarization with the adaptive threshold. Therefore, it was judged that binarization with the adaptive threshold was effective. The image subjected to binarization processing may include small dots of white and black. Morphological transformation processing of contraction and expansion was performed to remove noise, and small points were excluded.

A labeling process of connected components is performed as a method of extracting object parts from images. For each object portion whose width and height are the determination target size, each image of a predetermined image size is identified centering on the center of gravity of the object. The size to be determined is determined by the size of the target plankton. The 
five target plankton types to be differentiated are shown in Table 2. The lower limit of the classification target size is $80 \%$ of the width of Ha which is $10 \mu \mathrm{m}$, and the upper limit is $107 \%$ of the width of Ca which is $120 \mu \mathrm{m}$.

When the seawater is photographed with a microscope, even if the plankton appears in the photographed image, it is unclear because it is not in focus, and the type cannot be classified correctly in many cases. Therefore, it was decided to exclude the unclear images from the classification target before identification. A first-derivative filter was used as a determination method for an unclear image. The first-derivative filter is often used for edge detection of an image, but can also be used for the determination of unclear or blurred images. ${ }^{(12)}$ Figure 7 shows the determination of unclear images using a Sobel filter, which is a first-derivative filters. Figure 7(a) is an unclear or blurred image, Fig. 7(b) is a clear image; on the left is the input image, and on the right is an image representing the output result of the Sobel filter on the basis of brightness.

Figures 7(c) and 7(d) show the brightness of the cross section where the value after applying the Sobel filter to images in Figs. 7(a) and 7(b) is maximum. The dotted lines indicate the brightness of the input image, and the solid lines indicate the brightness of the image after using the Sobel filter. When the images after Sobel filtering are compared, the clear image has a higher brightness value, as shown in Fig. 7(d). It was confirmed that the Sobel filter could be an indicator of the degree of blurriness, as the values differ between the blurred image and the clear image. After classifying the image in accordance with the maximum value after Sobel filtering, the threshold value of the blurred image is determined by visual observation, and the image with the maximum value after Sobel filtering of 70 or less is determined to be a blurred image.

Table 2

(Color online) Image recognition target plankton.

\begin{tabular}{|c|c|c|c|}
\hline Harmful plankton & Abbreviation & Image sample & Number of images \\
\hline Chattonella antiqua & $\mathrm{Ca}$ & & $\begin{array}{c}\text { Training: } 27200 \\
\text { Validation: } 6400 \\
\text { Test: } 3200\end{array}$ \\
\hline Cochlodinium sp. type-Kasasa & $\mathrm{Ck}$ & $\theta$ & $\begin{array}{c}\text { Training: } 27200 \\
\text { Validation: } 6400 \\
\text { Test: } 3200\end{array}$ \\
\hline Chattonella marina & $\mathrm{Cm}$ & $\theta$ & $\begin{array}{c}\text { Training: } 27200 \\
\text { Validation: } 6400 \\
\text { Test: } 3200\end{array}$ \\
\hline Heterosigma akashiwo & $\mathrm{Ha}$ & • & $\begin{array}{c}\text { Training: } 27200 \\
\text { Validation: } 6400 \\
\text { Test: } 3200\end{array}$ \\
\hline Karenia mikimotoi & $\mathrm{Km}$ & 2 & $\begin{array}{c}\text { Training: } 27200 \\
\text { Validation: } 6400 \\
\text { Test: } 3200\end{array}$ \\
\hline
\end{tabular}




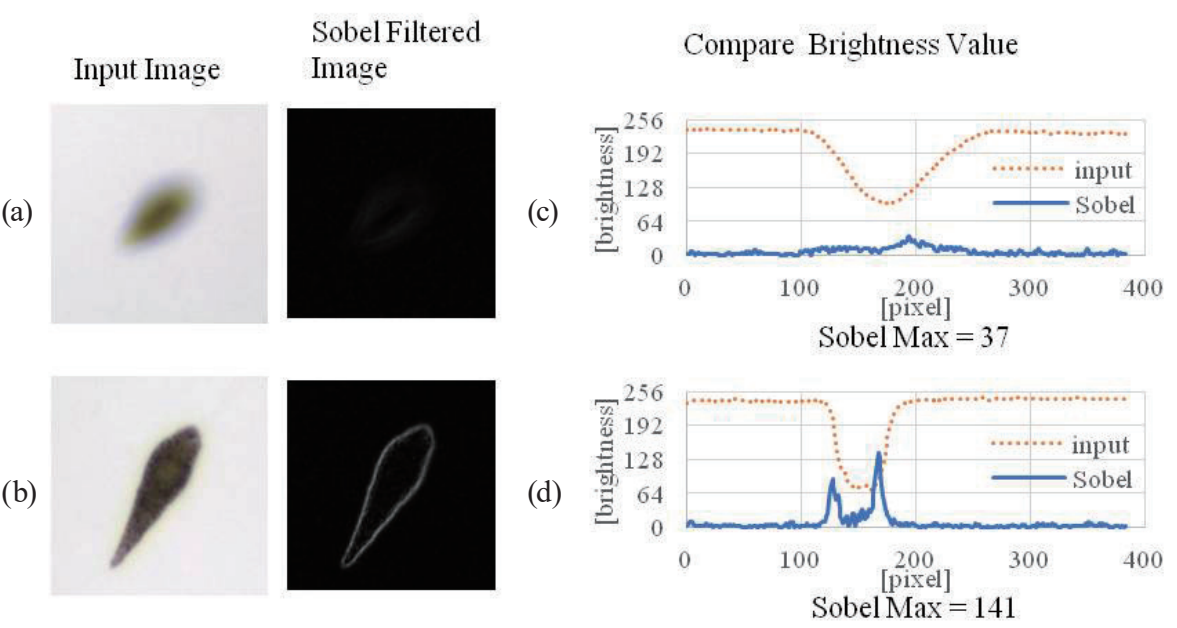

Fig. 7. (Color online) A blurred image and a clear image obtained with a Sobel filter.

\subsection{CNN model of plankton types classification}

We classify five types of plankton with a CNN. The input of CNN images are obtained by resizing the identified images shown in the previous section to $64 \times 64$ pixels. The output of the $\mathrm{CNN}$ is the classification result of the input image, which is the plankton type with the highest degree of accuracy among the five types of plankton and the accuracy value. Table 2 shows five types of plankton to be identified. When the CNN output accuracy is less than $70 \%$, it is classified as "Other".

The configuration of the CNN is shown in Fig. 8 (Convolution $\times 2-$ Activation Function ReLU - Max Pooling - Convolution $\times 2$ - Activation Function ReLU - Max Pooling - Flatten Fully Connect $\times 2-$ Activation Function Sigmoid). The activation function of the output layer is the Sigmoid function. The Softmax function is often used in image classification. However, in our system, when actual seawater is input, images other than the five types to be classified are input. By using not the Softmax function but the Sigmoid function, our system can classify "Other" type. Because the five output results of the output layer by the Sigmoid function take values independently, when images other than the five types learned are input, all the five output results take low values. Therefore, "Other" types different from the five types can be determined.

In order to classify plankton images using the $\mathrm{CNN}, \mathrm{CNN}$ parameter adjustment is performed by learning. Table 2 shows the images used as learning data for the CNN and the number of images used. The plankton of HAB cultured at Nagasaki Prefectural Institute of Fisheries is photographed with a camera connected to a microscope, and the learning data is selected by a human operator by visual observation. In identified and cut-out images, relatively clear images are used and images with blurriness of such an extent that classification is difficult to classify are not used as learning data. To improve learning accuracy, each image is rotated $\left(0^{\circ}\right.$, $45^{\circ}, 90^{\circ}, 135^{\circ}, 180^{\circ}, 225^{\circ}, 270^{\circ}$, and $315^{\circ}$ ) and the amount of image data is augmented. 


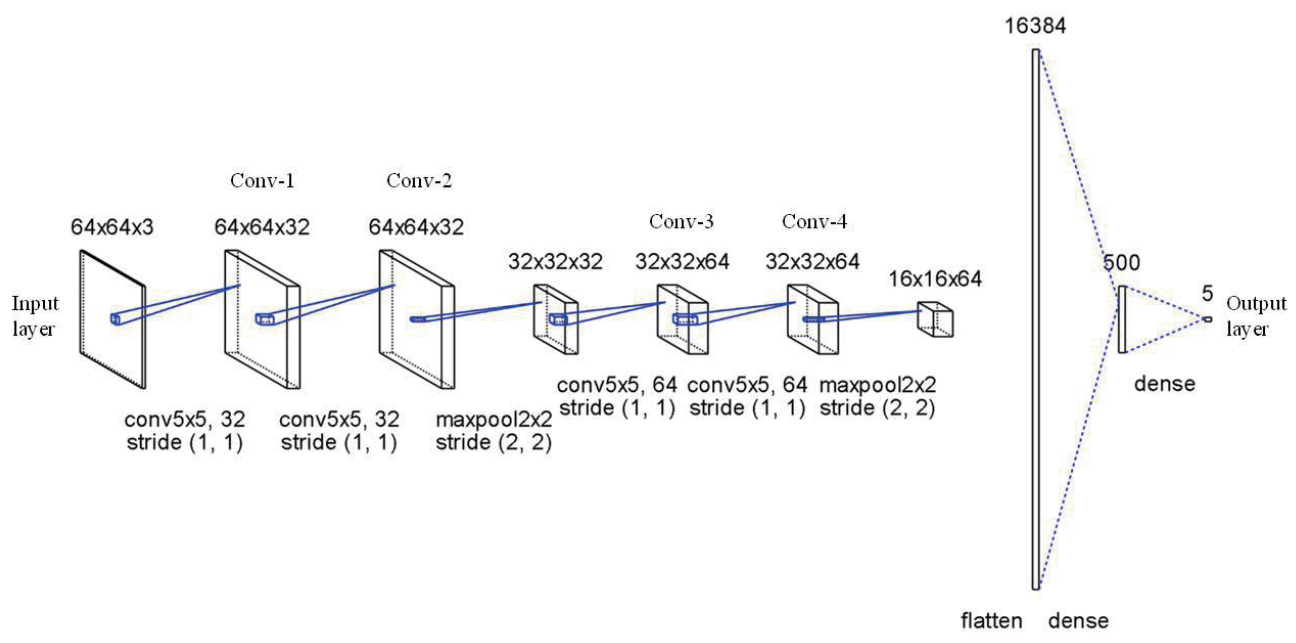

Fig. 8. (Color online) $\mathrm{CNN}$ configuration.

The curve of the CNN's learning process is shown in Fig. 9. The stochastic gradient descent (SGD) method is used as an optimization method in learning. After ten epochs, the increase in the accuracy rate tapers off, indicating that learning is completed. After the completion of learning, the accuracy rate based on test data evaluation is $99.5 \%$.

\subsection{Cell counting and HAB detection}

The method of counting the number of plankton cells in the image extracted from the microscope image is described below. After determining the sample area on the microscope where plankton is present, ten images are taken continuously (shooting interval: $150 \mathrm{~ms}$ ). The number of cells of each plankton type is counted for each image, and the maximum value of the number of cells of each plankton type amongst the ten images is taken as the number of cells of plankton in the sample. The reason for using the maximum value is that it is desirable to count as many harmful plankton cells as possible, to minimize the number of overlooked plankton cells. The area of observation is shifted, and the cell numbers at ten measuring points within a $0.1 \mathrm{~mL}$ plankton counter plate are summed and used for the determination of HAB detection. The overall sample volume is $0.3 \mathrm{~mL}$, of which $0.1 \mathrm{~mL}$ is used and within its area 10 points that clearly contain plankton are selected for analysis.

The formula for calculating the number of plankton cells and the HAB detection reference values are described below.

(1) Multiple images are acquired at each measuring point, and the maximum number of cells for each plankton type counted in each image is acquired. The number of cells of each plankton type at each measuring point is calculated as

$$
M_{i j}=\max \left(C_{i j 0}, C_{i j 1}, \cdots, C_{i j k}\right),
$$




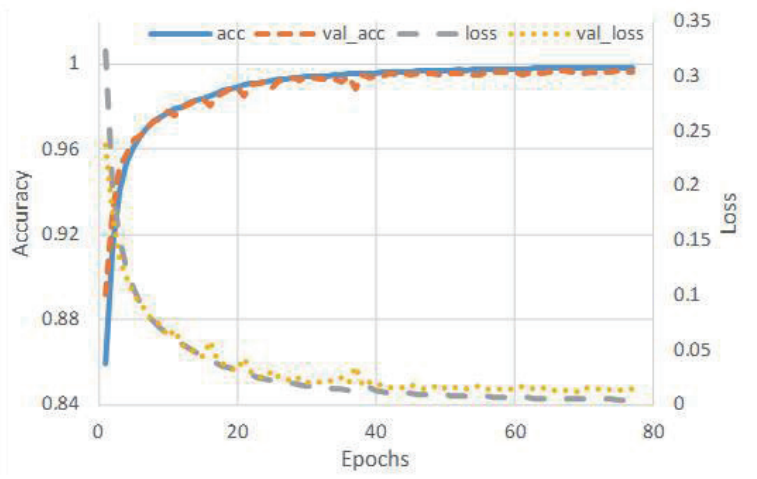

Fig. 9. (Color online) Learning process curve.

where $M$ is the number of cells of each plankton type at each measuring point and $C$ is the number of cells of each plankton type in each image. The suffix $i$ represents the plankton type, $j$ represents the measuring point and $k$ represents each image.

(2) The maximum number of cells of each plankton type at each measuring point is summed to obtain the total number of cells over the entire measuring area. The number of cells of each plankton type at all measuring points is calculated as

$$
S_{i}=\sum\left(M_{i 1}, M_{i 2}, \cdots, M_{i j}\right),
$$

where $S$ is the total number of cells of each plankton type over all measuring points and $M$ is the number of cells of each plankton type at each measuring point. The suffix $i$ represents the plankton type and $j$ represents the measuring point.

(3) The total number of cells at all measuring points is divided by the volume of seawater to be observed to obtain the density (number of cells per $1 \mathrm{~mL}$ ). The density (number of cells per $1 \mathrm{~mL}$ ) of each plankton type is calculated as

$$
D_{i}=\frac{S_{i}}{V},
$$

where $D$ is the density of plankton (number of cells per $1 \mathrm{~mL}$ ), $S$ is the total number of cells of each plankton type over all measuring points, and $V$ is the volume of seawater to be observed. The suffix $i$ represents the plankton type.

The following describes how HAB is determined. The density based on the counted results is compared with a reference density for each plankton, shown in Table 3 , to determine the risk level of a HAB. ${ }^{(13)}$ The risk level is ranked as alert or caution. Alert means it is recommended to stop feeding the cultured fish and to move the aquaculture cages to a place where the probability of HAB occurrence is presumed to be low. Caution means aquaculture fishermen should be aware of the plankton trends and to be prepared to stop feeding or to move aquaculture cages. These results are saved in a cloud database and used for the real-time notification described in Sect. 4. 
Table 3

$\mathrm{HAB}$ detection reference values.

\begin{tabular}{lccc}
\hline \multirow{2}{*}{ Harmful plankton } & \multirow{2}{*}{ Abbreviation } & \multicolumn{2}{c}{ Reference values (cells/mL) } \\
\cline { 3 - 4 } & & Alert & Caution \\
\hline Chattonella antiqua & $\mathrm{Ca}$ & 1 & 0.1 \\
Cochlodinium sp. type-Kasasa & $\mathrm{Ck}$ & 50 & 5 \\
Chattonella marina & $\mathrm{Cm}$ & 1 & 0.1 \\
Heterosigma akashiwo & $\mathrm{Ha}$ & 1000 & 100 \\
Karenia mikimotoi & $\mathrm{Km}$ & 50 & 10 \\
\hline
\end{tabular}

\subsection{Evaluation method of classification accuracy rate}

The evaluation of the accuracy rate of image recognition in the HAB detection system is described below. Images of HAB plankton cultured at the Nagasaki Prefectural Institute of Fisheries are taken with a microscope. 500 images of each of the five types of plankton, a total of 2500 images, were used as input images. As a result of the processing described in Sects. 3.2 to 3.4, the accuracy of the plankton counting results was used for the evaluation of the image recognition accuracy. The accuracy rate for each plankton type was calculated using Eq. (4), which excludes "Other" type. The image for evaluation contains unclearly observed plankton which is not in focus, and is classified as "Other" type because it could not be classified into any of the five types of plankton targeted. The classification of "Other" type is excluded from the calculation of the accuracy rate because it is considered not to be incorrect nor correct. The average accuracy rate of all plankton classification was calculated by Eq. (5).

$$
A_{i}=\frac{T_{i}}{U_{i}-X_{i}}
$$

where $A$ is the accuracy of plankton classification, $T$ is the number of plankton cells correctly classified, $U$ is the number of all plankton cells, and $X$ is the number of plankton cells classified as "Other". The suffix $i$ represents the plankton type.

$$
A v=\operatorname{Average}\left(A_{1}, \cdots, A_{i}\right)
$$

$A v$ is the average accuracy of classification and $A$ is the individual accuracy of classification. The suffix $i$ represents the plankton type.

\section{Real-time Notification System}

In this section, we describe a real-time notification system that notifies the relevant people, such as fishermen, of the results of the HAB detection system. The system has three functions; an email notification function of the HAB classification result, a display function on a map, and a display function of seas surface images. 


\subsection{Email notification of HAB detection}

Push notifications of HAB detection system information updates are sent to the relevant people. The system classifies the result as one of three levels (normal, caution, and alert), and sends notification only for caution and alert levels. The notification email is automatically delivered to the email address of the people registered in advance. In the email text, the date and time of water sampling, location information, the URL of the web application, and results of counting harmful plankton for each water sampling depth are detailed. By tapping the URL, it is possible to access information on the web application and obtain graphical information.

\subsection{Map display of HAB detection}

The web application, shown in Fig. 10, displays the amount of harmful plankton present in three levels on the map application. Markers indicating water sampling points are displayed in three colors $($ normal $=$ green, caution $=$ yellow, and alert $=$ red) so that the risk level can be understood at a glance. By tapping the marker on the map, more detailed information, such as harmful plankton counts for each depth at that point, appears. It is also possible to browse data retrospectively from the calendar UI. In addition, browsing of a sea surface photographs is possible by tapping the aerial photography image button.

\subsection{Display sea surface images}

The drone photography points are displayed by markers on the map application, as shown in Fig. 11. By tapping one of the markers, the image taken from the drone is displayed. Fisheries personnel of concern usually notice anomalies in the color of the sea, and exchange information, enabling numbered color samples to be used in Nagasaki Prefecture. Among them, 7 colors
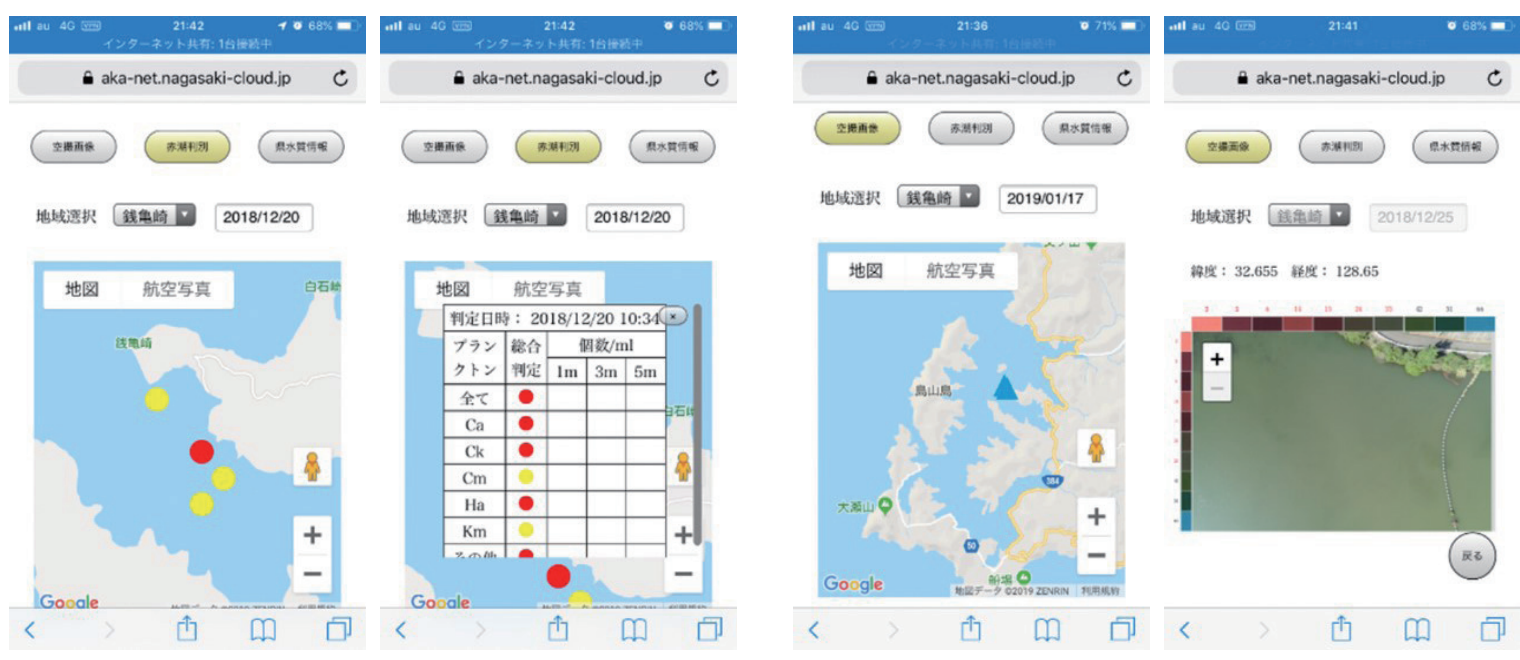

Fig. 10. (Color online) Display of results on a map.

Fig. 11. (Color online) Display of sea surface image. 
indicating abnormal and 3 colors indicating normal are often used, and the color bars of the color samples are placed along the top and the left side of the captured image for comparison with the sea surface image. The captured image can be scrolled and zoomed, and can be easily compared with the color samples.

\section{Results and Discussion}

\subsection{Evaluation of classification accuracy of HAB detection system}

The image recognition result in the HAB detection system was evaluated using Eqs. (4) and (5) in Sect. 3.5. The evaluation results are shown in Table 4. The upper part of Table 4 shows the numbers of plankton cells predicted using the input image of each plankton type. Shaded portions indicate the number of matches between input and predicted results. The lower part of Table 4 shows the individual accuracy rate and the average accuracy rate of each plankton classification; and the average accuracy rate was $92.2 \%$. The test result at the completion of the learning of the CNN described in Sect. 3.3 is $99.5 \%$. There is a difference between the accuracy rate at the learning stage and at the evaluation stage. The most significant difference between learning and evaluation is the final classification output from the CNN. At the evaluation stage, 10 images taken continuously were used as a set for classification, and each image is classified as "Other" when the output of $\mathrm{CNN}$ is less than $70 \%$. These condition differences cause that the number of correct answers decreases and the number of incorrect answers increases, so it is considered that the accuracy rate at the evaluation stage is lower than at the learning stage.

In addition, in the evaluation results, $\mathrm{Ca}$ and $\mathrm{Cm}$ are often classified incorrectly. $\mathrm{Ca}$ tends to have a longer tail and its body is longer than that of $\mathrm{Cm}$, but $\mathrm{Ca}$ and $\mathrm{Cm}$ are of the same Chattonella genus, morphologically similar, and sometimes can be difficult to distinguish even by visual observation. However, although these two could not be clearly classified here, since both present the same level of risk as harmful plankton, it seems that no major problem will arise even if the two are confused. In the future, it may be possible to improve the performance using time-series analysis considering the movement of the plankton.

Table 4

Evaluation results of image classification.

\begin{tabular}{llrrrrc}
\hline \multirow{2}{*}{ CNN Model Classification Results } & \multicolumn{3}{c}{ Correct } \\
\cline { 2 - 6 } & $\mathrm{Ca}$ & $\mathrm{Ca}$ & $\mathrm{Ck}$ & $\mathrm{Cm}$ & $\mathrm{Ha}$ & $\mathrm{Km}$ \\
\hline \multirow{6}{*}{ Prediction (Count) } & $\mathrm{Ck}$ & 0 & 65 & 4 & 0 & 0 \\
& $\mathrm{Cm}$ & 25 & 1 & 163 & 0 & 0 \\
& $\mathrm{Ha}$ & 0 & 0 & 0 & 167 & 0 \\
& $\mathrm{Km}$ & 2 & 0 & 1 & 7 & 64 \\
\hline \multirow{2}{*}{ Accuracy (Other excluded) (\%) } & Other & 43 & 3 & 16 & 5 & 2 \\
\hline
\end{tabular}


Table 5

Required time for the proposed and conventional methods.

\begin{tabular}{lcccccc}
\hline & Sampling & Store & Transport & Analysis & Notification & Total \\
\hline Conventional method & $1 \mathrm{~h}$ & $3 \mathrm{~h}$ & $30 \mathrm{~min}$ & $1 \mathrm{~h}$ & $30 \mathrm{~min}$ & $\mathbf{6 ~ h}$ \\
Proposed method & $6 \mathrm{~min}$ & $0 \mathrm{~h}$ & $0 \mathrm{~min}$ & $4 \mathrm{~min}$ & $20 \mathrm{~s}$ & $\mathbf{1 0 . 3} \mathbf{~ m i n}$ \\
\hline
\end{tabular}

\subsection{Questionnaire on satisfaction level of real time notification}

A questionnaire was conducted to evaluate the notification function of the application. Six answers were obtained from aquaculture companies and fishery cooperatives. On a 5-level rating with 5 as the maximum and 1 as the minimum, the average ratings are described below. The "ease of use" (Q.1) is 4.5, "intuitively understood" (Q.2) is 4.3, and "useful for business" (Q.3) is 4.5. From Q.4 "How could this system be improved or what other functions could be included to improve the system", we confirmed opinions regarding tidal current prediction after the occurrence of the algal bloom, the mechanism of notifications from the aquaculture company side, and the prevention of reflection, which hinder the judgment of the color of the sea surface.

\subsection{Comparison of required time with conventional methods}

The effect of the efficiency improvement in this system was evaluated by comparing the required time with the conventional method. The evaluation results are shown in Table 5. It indicated that all items resulted in reducing the time required, and the total process time was shortened from $6 \mathrm{~h}$ to 10.3 minutes. The goal of reducing the process time was achieved.

\section{Conclusions}

In this study, we developed a system to determine HABs in a shorter time than the conventional method and verified its effectiveness. In the developed system, patrolling the sea surface and sampling seawater by drone, detection of HAB from microscopic images, automatic notification by email and web application are carried out. We verified that this system enables early detection and early countermeasures against HABs in a shorter time than the conventional method.

Future issues we are considering in relation to this study are as follows. In this study, plankton cultured at the Nagasaki Prefectural Institute of Fisheries was used as the basis of the classification of plankton type. However, the validation of plankton samples taken from the HAB-affected sea areas is more desirable. Taking the feedback from aquaculture fishermen regarding this system into account will be essential for improvement. We will endeavor to make this system more effective for aquaculture fishermen by implementing the observation of sea conditions by setting up fixed point observation data buoys and using satellite data and the prediction of the movement of algal blooms using tidal current movements. 


\section{Acknowledgments}

This work was supported by Project for IoT Services Creation of the Ministry of Internal Affairs and Communications in Japan. The authors wish to express their appreciation to Dr. Toshifumi Yamatogi and Mr. Sou Hirae (Nagasaki Prefectural Institute of Fisheries) who provided the cultured HAB plankton and the necessary laboratory equipment. We are grateful to the people of Goto-shi for their cooperation. We would like to thank Mr. Shouji Nakajima (Ryokeiso Corporation) and Mr. Yasunori Izumi (Musou Kagaku Corporation) for their integral contribution to this development. On the basis of our results, further research will be promoted with the support of the Strategic Information and Communications R\&D Promotion Programme (SCOPE) of the Ministry of Internal Affairs and Communications in Japan.

\section{References}

1 United Nations, Department of Economic and Social Affairs, Population Division: World Population Prospects: The 2017 Revision, Key Findings and Advance Tables. Working Paper No. ESA/P/WP/248 (2017).

2 Food and Agriculture Organization of the United Nations: The State of World Fisheries and Aquaculture 2018 - Meeting the sustainable development goals (2018).

3 J. H. W. Lee, I. J. Hodgkiss, K. T. M. Wong, and I. H. Y. Lam: Estuarine, Coastal and Shelf Science 65 (2005) 172-190. https://doi.org/10.1016/j.ecss.2005.06.005

4 R. Elkadiri, C. Manche, M. Sultan, A. Al-Dousari, S. Uddin, K. Chouinard, and A. Z. Abotalib: IEEE J. Sel. Top. Appl. Earth Obs. Remote Sens. 9 ( 2016) 11. https://doi.org/10.1109/JSTARS.2016.2555898

5 I. Yamamoto: Proc. 6th Int. Conf. Aerospace and Aerodynamics (J. Aeronaut Aerospace Eng., 2018). https:// doi.org/10.4172/2168-9792-C2-024

6 C. Koparan, A. B. Koc, C. V. Privette, C. B. Sawyer, and J. L. Sharp: Water 10(5) (2018) 655. https://doi. org $/ 10.3390 /$ w10050655

7 Q. Hu and C. Davis: Estuarine, Marine Ecology Progress Series Vol. 295 (2005) 21-31.

8 A. Zheng and M. Wang: Convolutional Neural Network-based Plankton Image Classification System (2015).

9 C. K. Chow and T. Kaneko: Computers and Biomedical Research 5 (1972) 4. https://doi.org/10.1016/00104809(72)90070-5

10 K. Bhargavi and S. Jyothi: International Journal of Innovative Research \& Development 3 (2014) 12.

11 M. Chandrakala: International Journal of Engineering Trends and Technology 35 (2016) 9. https://doi. org/10.14445/22315381/IJETT-V35P285

12 J. L. Pech-Pacheco, G. Cristobal, J. Chamorro-Martinez, and J. Fernandez-Valdivia: Proc. 15th Int. Conf. Pattern Recognition. ICPR-2000 (IEEE, 2000). http://doi.org/10.1109/ICPR.2000.903548

13 T. Yamatogi and N. Ishida: Occurrence of fishery damage due to red tide of low cell density in islands sea area of Nagasaki Prefecture: Advances in Harmful Algae Bloom Research (Kouseisha-kouseikaku, Tokyo, 2016) p. 138. (in Japanese)

\section{About the Authors}

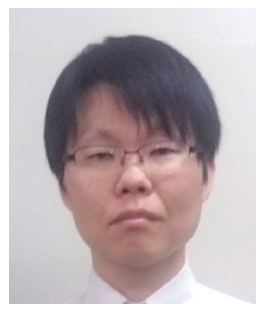

Fukuyoshi Kimura received his B.S. degree in engineering science from Osaka University, Japan, in 2002. He joined System Five Corporation in 2005. Since then, he has been engaged in computer system development such as training simulators for thermal power plants and database systems for medical research. (f-kimura@sfk-nga.co.jp) 


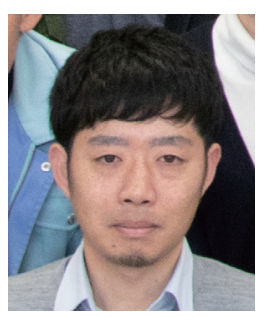

Akihiro Morinaga received his Dr. Eng. degree in 2016 from Kyushu University, Japan and became an assistant professor at Nagasaki University in 2016. His current research interests include robotics, mechatronics and control theory.

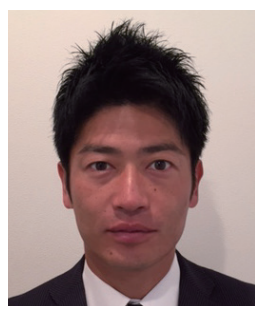

Masayoshi Fukushima received his associate degree from National Institute of Technology, Hachinohe College, Japan, in 1998. He joined DDI Corporation in 1998. Since then, he has been engaged in the construction of corporate networks. He belonged to the KDDI Reconstruction Support Office in 2012. He was relocated to Higashi Matsushima and Higashimatsushima Organization for Progress and Economy, Education, Energy in 2013. Since 2017, he has been a manager at a Local Development Support Office, IoT Business Development Division. He has been engaged in reconstruction and local activation through IoT utilization.

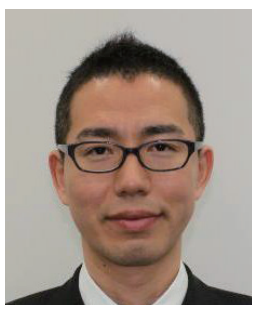

Tomonari Ishiguro received his B.A. degree from Tohoku University, Japan, in 2001. He joined KDDI Corporation in 2001. Since then, he has been engaged in customer service and human resources work. He belonged to the KDDI Reconstruction Support Office and was relocated to Kamaishi in 2012. Since 2017, he has been a manager at a Local Development Support Office, IoT Business Development Division. He has been engaged in reconstruction and local activation through IoT utilization.

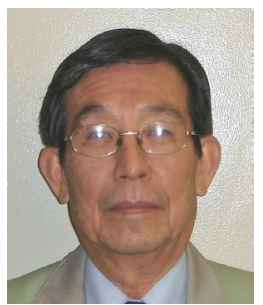

Yasuhiko Sato graduated from Nagasaki Prefectural Nagasaki Technical High School, Japan, in 1963. He worked for Mitsubishi Heavy Industry from 1963 to 1992 . He has been engaged in the research of measurement and control and the development of computer application products. In 1994, he established System Five Corporation and became president and representative director. He has been engaged in the development of technical systems.

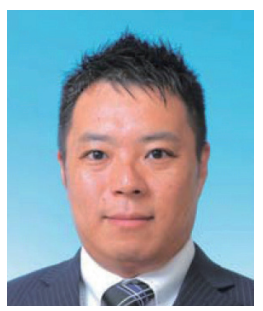

Akihiro Sakaguchi received his Ph.D from Hiroshima University in 2006. He has been an associate professor in the Department of Control Engineering at the National Institute of Technology, Sasebo college. His current research interests are in the area of grinding technology and image measurement. $\mathrm{He}$ is a member of the Japan Society for Abrasive Technology and the Japan Society for Precision Engineering. 


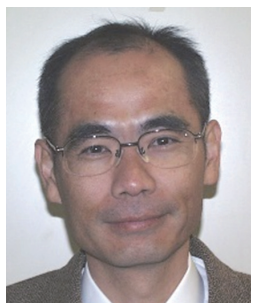

Tomoyuki Kawashita received his Ph.D in engineering from the University of Kumamoto, Japan, in 1994. He worked for Yaskawa Electric Corporation from 1983 to 1991. He is now a professor at National Institute of Technology, Sasebo College. His current research interests include mechatronics, image processing, and abrasive processing.

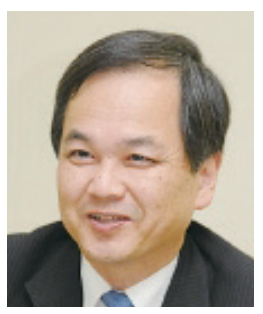

Ikuo Yamamoto received his Dr. Eng. degree in 1994 from Kyushu University, Japan, and became a professor at Kyushu University in 2005, University of Kitakyushu in 2007, and Nagasaki University, Japan, in 2013. He also became a GlobalScot member of Scotland, UK, in 2017. His current research interests include robotics, mechatronics, and IoT.

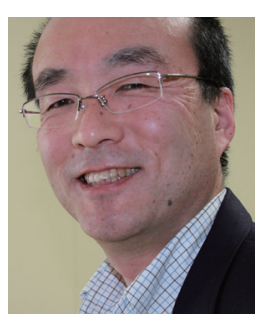

Toru Kobayashi received his Ph.D in engineering from the University of Electro-Communications, Japan, in 2011. He worked for NTT from 1987 to 2013. He is a senior member of the IEEE Computer Society, IPSJ, and IEICE. $\mathrm{He}$ is currently a professor in the Division of Electrical Engineering and Computer Science, Graduate School of Engineering, Nagasaki University. 